\title{
Current status and issues of uniportal video-assisted thoracic surgery in Japan
}

\author{
Kyoji Hirai ${ }^{1}$, Jitsuo Usuda ${ }^{2}$ \\ ${ }^{1}$ Department of Thoracic Surgery, Nippon Medical School Chiba Hokusoh Hospital, Inzai, Chiba, Japan; ${ }^{2}$ Division of Thoracic Surgery, Nippon \\ Medical School, Tokyo, Japan \\ Contributions: (I) Conception and design: Both authors; (II) Administrative support: None; (III) Provision of study materials or patients: K Hirai; \\ (IV) Collection and assembly of data: K Hirai; (V) Data analysis and interpretation: Both authors; (VI) Manuscript writing: Both authors; (VII) Final \\ approval of manuscript: Both authors. \\ Correspondence to: Kyoji Hirai, MD. Department of Thoracic Surgery, Nippon Medical School Chiba Hokusoh Hospital, 1715 Kamakari, Inzai, \\ Chiba 270-1674, Japan. Email: ky-hirai@nms.ac.jp.
}

Background: Uniportal video-assisted thoracic surgery (U-VATS) is performed routinely in some countries for respiratory diseases including lung cancer. Several studies report both advantages and disadvantages of the procedure. We review the history of U-VATS in Japan, highlight limitations of the procedure, and discuss the current status and surgical outcomes of U-VATS for anatomical lung resections performed at our institution for patients with early-stage cancer.

Methods: We performed a retrospective review of clinical factors and surgical outcomes in patients with clinical stage I non-small-cell lung cancer who underwent surgery at our institution between 2013 and 2019. We also present representative videos of the procedure performed at our institution and review limitations of the procedure as it becomes more prevalent in Japan.

Results: A total of 152 lobectomies and 33 segmentectomies were performed for clinical stage I non-smallcell lung cancer during the study period. Lobectomy and segmentectomy had a mean operative time of $145 \pm 19$ and $114 \pm 17 \mathrm{~min}$, intraoperative blood loss of $72 \pm 11$ and $52 \pm 9 \mathrm{~mL}$, drainage for $1.9 \pm 0.8$ and $2.1 \pm 0.7$ days, hospital length of stay of $7.8 \pm 1.4$ and $6.8 \pm 1.1$ days, and the number of $17 \pm 3$ and $14 \pm 4$ lymph nodes (LNs) were dissected, respectively. $5.9 \%$ and $3.0 \%$ of patients underwent conversion to thoracotomy in lobectomy group (LG) and segmentectomy group (SG), respectively. A total of 14 cases developed the complication (12 vs. 2 in LG and SG, respectively). There was no mortality in either group. The average of numeric rating scale (NRS) on postoperative day 30 was 2.1 and 2.4 for lobectomy and segmentectomy, respectively. The 5 -year overall survival (OS) rate was $80.1 \%$ in LG and the 4-year progression-free survival (PFS) rate of segmentectomy was $94.1 \%$.

Conclusions: We demonstrated that the outcomes of U-VATS for anatomical lung resections in patients with early-stage lung cancer was within permissible range. Our findings suggest that U-VATS anatomical lung resection may be a suitable minimally invasive option to achieve preferable treatment outcomes.

Keywords: Uniportal video-assisted thoracic surgery (U-VATS); multiport video-assisted thoracic surgery (M-VATS); minimally invasive surgery

Received: 03 January 2020; Accepted: 03 February 2020; Published: 05 July 2020.

doi: 10.21037 /jovs.2020.02.07

View this article at: http://dx.doi.org/10.21037/jovs.2020.02.07 


\section{Introduction}

Uniportal video-assisted thoracic surgery (U-VATS) has become prevalent among thoracic surgeons in a part of countries including Japan (1-3). U-VATS and roboticassisted thoracoscopic surgery (RATS) are two types of minimally invasive surgical techniques. Uniportal procedures are feasible although they can be technically challenging. RATS is superior to U-VATS in terms of the ability to perform procedures that require fine motor skills and to maintain wide visual fields during the procedure; however, it is not as feasible and more costly compared with U-VATS. U-VATS is also considered safer as it is easier than RATS to be converted to thoracotomy when needed. Thus, suitable procedures should be selected to meet the needs of specific cases. In the present study, we present representative videos of the procedure and review the treatment outcomes of U-VATS for anatomical lung resection that were performed at our institution for patients with early-stage lung cancer.

\section{Methods}

A retrospective review of a prospectively maintained database identified 212 patients with clinical stage I non-small-cell lung cancer who underwent curative thoracoscopic surgery at Nippon Medical Chiba Hokusoh Hospital between June 2012 and March 2019. One hundred and fifty-two of lobectomies and 33 of segmentectomies were performed for the patients with clinical stage I lung cancer by the same thoracic surgeon. Our indications for lung segmentectomy included peripheral clinical T1aN0M0 non-small lung cancer with a tumor less than $2 \mathrm{~cm}$ in diameter and a ground-glass lesion showing a solid part of less than $50 \%$ (4). In identifying the intersegmental lines, inflate-deflate method using 6- or 8-Fr pediatricsize bladder catheter via peripheral bronchus was provided. The representative procedures of uniportal segmentectomy (right anterior segmentectomy) are shown in Video 1. As for segmentectomies, simple segmentectomies were performed. In U-VATS, a $3.5-5.0 \mathrm{~cm}$ incision was made on the anterior to middle axillary line, followed by the attachment of a Wrap Protector (Hakko Inc., Japan). Protective procedures to the intercostal nerves were provided by the surgeon and assistants. The procedure was atraumatically performed as much as possible in order to prevent contact between the thoracoscope and forceps and intercostal nerves. The thoracoscope was mostly handled at an angle of

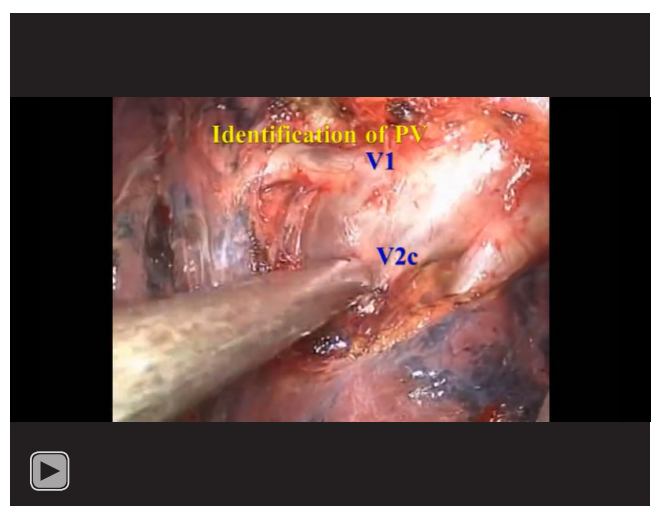

Video 1 Surgical technique of right S3 segmentectomy (5).

more than 45 degrees from the chest wall. Surgery was performed using a 10- or 5-mm 30-degree oblique-viewing thoracoscope and facing/inverted dual-monitors without any specific device. The Wrap Protector mini-mini was routinely used to open incisions, and a small rib retractor was not used. The energy device, HARMONIC scalpel (Ethicon, USA) was used for mediastinal lymph node (LN) dissection. Various surgical factors (operative outcomes), the incidence of complications, postoperative complications (prolonged air leak, atelectasis, empyema, wound infection), and 60-day mortality were evaluated. Postoperative wound pain was monitored using the numeric rating scale (NRS). NRS was evaluated on postoperative day 30. Prognostic evaluation such as progression-free survival (PFS) rate and overall survival (OS) rate using Kaplan-Meier analysis in both groups was carried out.

The study was conducted in accordance with the Declaration of Helsinki (as revised in 2013). The study was approved by the institutional review board and the committee of Nippon Medical Chiba Hokusoh Hospital (No. 616). Informed consent to use the data-use agreement including surgical video was obtained from all patients before surgery.

\section{Statistical analysis}

Quantitative variables are presented as medians $[ \pm$ standard deviation (SD)] or ranges where appropriate. Continuous variables were compared using the Mann-Whitney $U$ test where appropriate. The Kaplan-Meier method was used to visualize PFS and OS of segmentectomy group (SG) and lobectomy group (LG). All statistical analyses were performed with the software SPSS version 17 for Windows 
Table 1 Clinical characteristics of study population

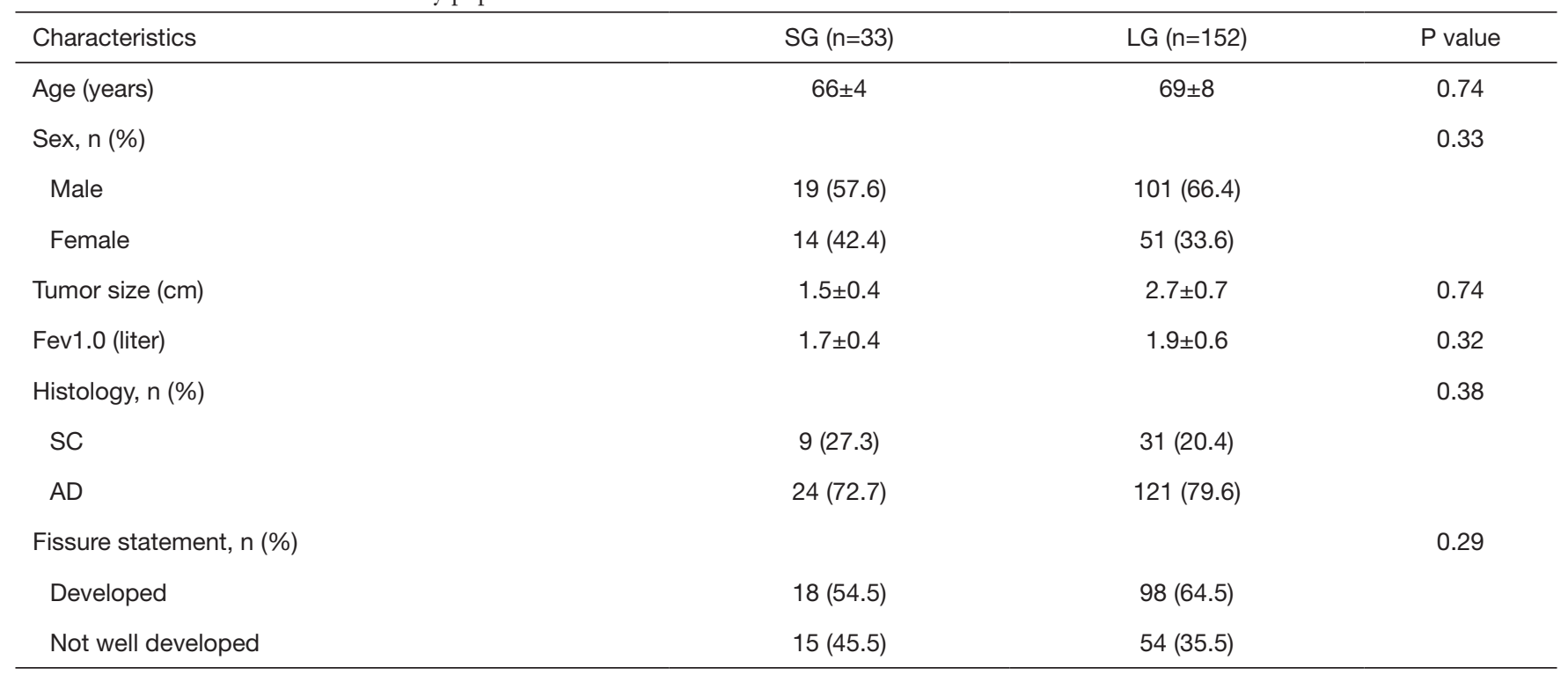

Data are presented as mean \pm SD or number (percentage). SD, standard deviation; SG, segmentectomy group; LG, lobectomy group; Fev 1.0 , forced expiratory volume in $1.0 \mathrm{~s}$; SC, squamous cell carcinoma; $\mathrm{AD}$, adenocarcinoma.

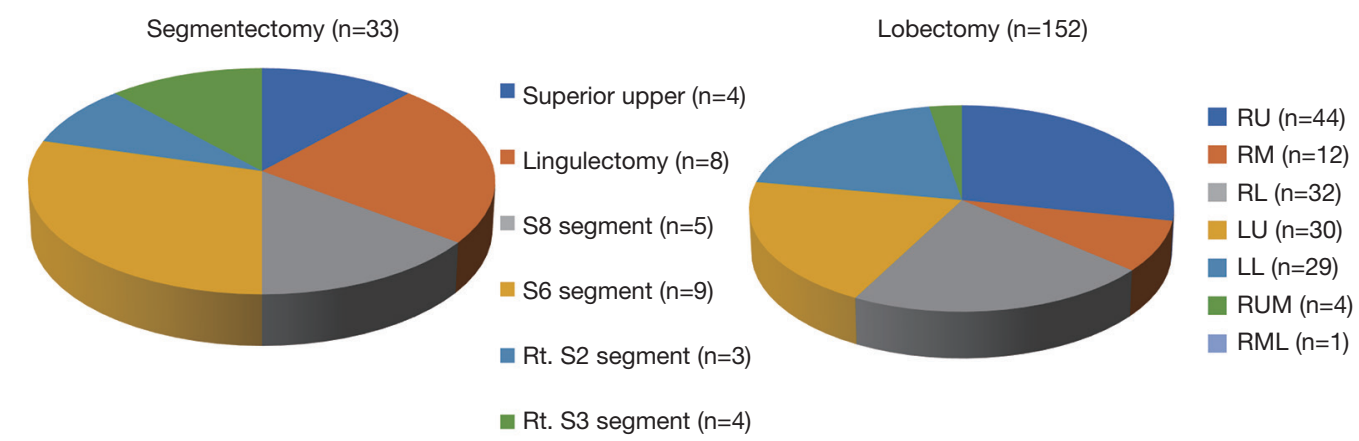

Figure 1 Distribution of segmentectomy and lobectomy in U-VATS. RU, right upper; RM, right middle; RL, right lower; LU, left upper; LL, left lower; RUM, right upper and middle; RML, right middle and lower.

(IBM Corp, Armonk, NY, USA). P values of $<0.05$ were considered to indicate significance for all parameters.

\section{Results}

\section{Preoperative data and resected lobe of lung}

From 2013 to 2019, we performed 152 lobectomies and 33 segmentectomies by U-VATS for clinical stage I lung cancer. The preoperative patient's characteristics are summarized in Table 1. As shown in Table 1, in LG and SG, age, sex, tumor size, forced expiratory volume $1.0 \mathrm{~s}$ (Fev 1.0), histology and fissure statement were indicated.

\section{Postoperative data}

The mean postoperative wound length in both groups was $4.2 \mathrm{~cm}$. Distribution of lobectomies in both groups is shown in Figure 1. The most of the lobectomies in LG was right upper (RU) lobectomies and S6 segmentectomy was the most in SG. The postoperative outcomes are shown in Table 2. The data of LG are shown as follows. The median operative time was $145 \pm 19 \mathrm{~min}$, and the median operative blood loss was $72 \pm 11 \mathrm{~mL}$. The mean number of dissected LNs was $17 \pm 3$. The mean duration of the drainage duration and postoperative hospital stay and were $1.9 \pm 0.8$ and $7.8 \pm 1.4$, respectively. In $\mathrm{SG}$, the median operative time 
Table 2 Postoperative outcomes

\begin{tabular}{|c|c|c|c|}
\hline Variables & $S G(n=33)$ & $L G(n=152)$ & $P$ value \\
\hline Blood loss (mL) & $52 \pm 9$ & $72 \pm 11$ & 0.44 \\
\hline The number of dissected LN & $14 \pm 4$ & $17 \pm 3$ & 0.47 \\
\hline Drainage duration (days) & $2.1 \pm 0.7$ & $1.9 \pm 0.8$ & 0.65 \\
\hline Conversion to thoracotomy (\%) & $1(3.0)$ & $9(5.9)$ & 0.81 \\
\hline NRS on POD 30 & $2.4 \pm 0.5$ & $2.1 \pm 0.9$ & 0.67 \\
\hline Morbidity, n (\%) & $2(6.1)$ & $12(7.9)$ & 0.98 \\
\hline Mortality, n (\%) & 0 & 0 & - \\
\hline
\end{tabular}

Data are presented as mean \pm SD or number (percentage). Postoperative complications were including with prolonged air leak more than 5 days after surgery, atelectasis, empyema and wound infection. SD, standard deviation; SG, segmentectomy group; LG, lobectomy group; LN, lymph node; NRS, numeric rating scale.

was $114 \pm 17 \mathrm{~min}$, and the median operative blood loss was $52 \pm 9 \mathrm{~mL}$. The mean number of dissected LNs was $14 \pm 4$. The mean duration of the drainage duration and postoperative hospital stay and were $2.1 \pm 0.7$ and $6.8 \pm 1.1$, respectively. With regard to the operation time, blood loss, the number of dissected LNs, drainage duration, hospital stays, conversion rate to thoracotomy, incidence of postoperative complications including 7 prolonged air leakages lasting more than 5 days, 3 atelectasis requiring bronchoscopic suction after operation, 2 empyema requiring prolonged drainage and 2 wound infection requiring drainage. No mortality was recorded in both groups. The average of NRS on postoperative day 30 was 2.1 in LG and 2.4 in SG. According to Kaplan-Meier analysis, the 5 -year OS rate and the 5 -year PFS rate was $80.1 \%$ and $78.5 \%$ in LG (Figure $2 A, B$ ) and was $100 \%$ and $94.1 \%$ (Figure 3) in SG.

\section{Discussion}

The recent consensus report from the Uniportal VATS Interest Group (UVIG) of the European Society of Thoracic Surgeons (ESTS) describes the procedures of U-VATS for lobectomy (6). The report was generated based on questionnaires from 31 thoracic surgeons in 18 countries. According to the report, U-VATS was typically performed with $<4 \mathrm{~cm}$ incision on the anterior axillary or middleanterior axillary line, with a surgical assistant standing on the same side as the primary surgeon. In terms of the indications for U-VATS, $65 \%$ of the surgeons considered
$\mathrm{T} 1$ and T2b stages to be appropriate for U-VATS and only $7 \%$ of them considered N0 to be a contraindication for U-VATS. Furthermore, only $3 \%$ of them considered that U-VATS should be contraindicated for patients at high risk of adhesion such as those with a history of pleurisy. Future studies on U-VATS should provide additional insights as to the characteristics of U-VATS. M-VATS is difficult to characterize since the specific procedures of M-VATS, such as the position and number of trocars, often vary across different institutions. On the other hand, the minimally invasive nature of U-VATS can be easily evaluated since there is only one incision made to the chest wall, thus facilitating the standardization of the procedure.

We have already performed over 200 cases of U-VATS for anatomical lung resections. Based on our experience, U-VATS is technically more suited than M-VATS for the resection of well-lobulated lungs. The technical procedures involved in U-VATS are more intuitive than M-VATS. Although there is some interreference with surgical instruments, procedural movements during U-VATS are similar to those performed during thoracotomy. In addition, some studies suggest that U-VATS may shorten the duration of RU lobectomies (7). Actually, in our study, there was no significant difference in regard with the operative outcomes of U-VATS anatomical lung resection. The conversion rates to thoracotomy were also within the permissible range. Especially as for converting U-VTAS lobectomy, we reviewed based on three reports including our data $(2,8,9)$. 

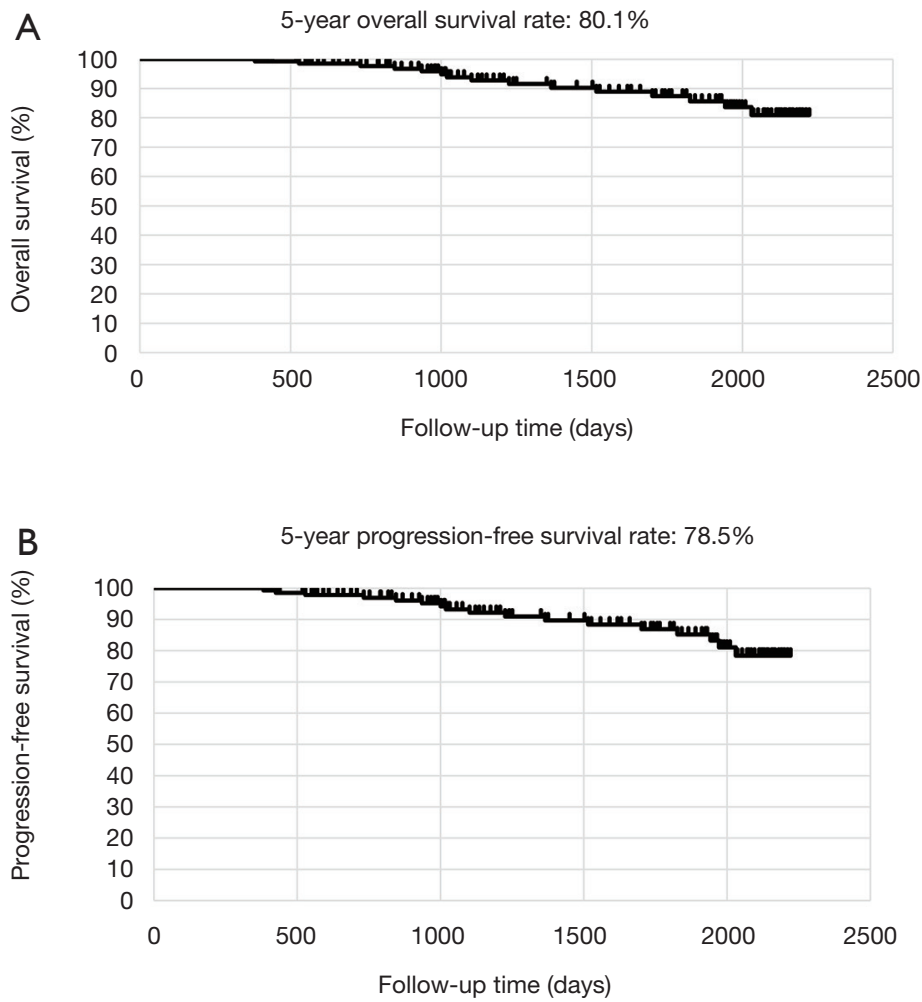

Figure 2 Kaplan-Meier analysis of OS and PFS after U-VATS lobectomy (n=152). The 5-year OS rate (A) and the 5-year PFS rate (B) in LG was $80.1 \%$ and $78.5 \%$. LG, lobectomy group; U-VATS, uniportal video-assisted thoracic surgery; OS, overall survival; PFS, progression-free survival.

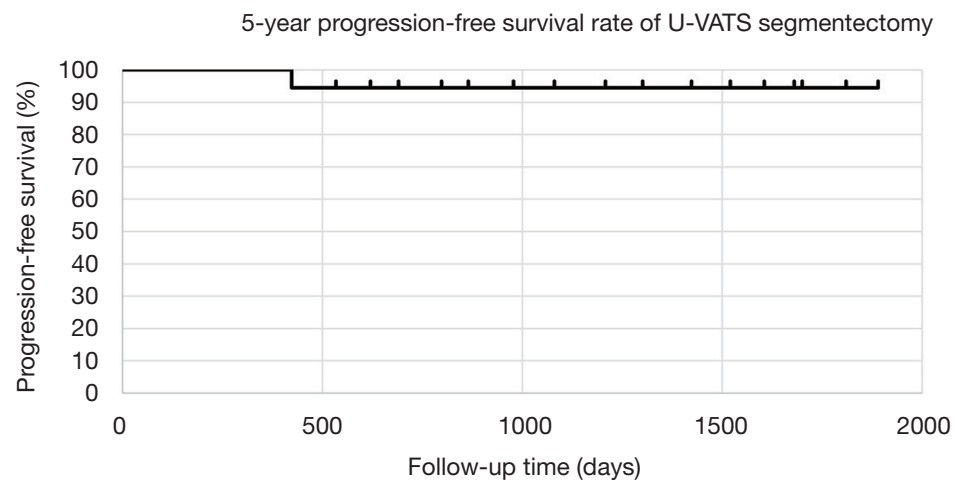

Figure 3 Kaplan-Meier analysis of PFS after U-VATS segmentectomy (n=33). The 5-year PFS rate in SG was $94.1 \%$. SG, segmentectomy group; U-VATS, uniportal video-assisted thoracic surgery; PFS, progression-free survival.

Notably, U-VATS is associated with a low risk of postthoracotomy pain syndrome that can occur in some patients and typically lasts for over 2 months. We previously performed a retrospective study and demonstrated that the rate of post-thoracotomy pain syndrome was lower in patients who underwent U-VATS compared to those who underwent M-VATS (10). Acute pain is often caused by the presence of a drain in the surgical site, and typically resolves after the removal of the drain. Patients with narrower intercostal spaces tend to be at a higher risk of post- 
thoracotomy pain syndrome, and may require conversion to the dual portal procedure to avoid contact between surgical instruments and the intercostal nerves.

Currently, U-VATS is most commonly performed in China. In contrast to RATS, U-VATS can be performed within a shorter period of time, allowing to fit in 3-4 surgical cases for lung cancer in a day. Given its convenience, U-VATS is likely more optimal in terms of health care efficiency for early-stage cancer. RATS is likely more suited for cases with a narrow surgical field that require superior motor skills. In thoracic surgery, these cases may include angioplasty, bronchoplasty, and LN dissection. In fact, a study demonstrated that RATS had a superior propensity score than U-VATS in terms of the number of LNs dissected (11). We anticipate that an increasing number of workshops for U-VATS will be held in Japan, and consequently, U-VATS will become more common in institutions that do not have or have the capacity to use surgical robots.

In this report, clinical data and prognostic evaluation are originated from single center and the same surgeon operated all patients for approximately 6 years. In our experience, compared to M-VATS, U-VATS has some disadvantages in terms of surgical quality in present, however if surgeons blush up their techniques much more, this procedures will be refined and common in thoracic surgeons considering the history that spread M-VATS over the world previously.

\section{Acknowledgments}

Funding: None.

\section{Footnote}

Provenance and Peer Review: This article was commissioned by the Guest Editor (Meinoshin Okumura) for the series "Dedicated to the 36th Annual Conference of Japanese Association for Chest Surgery" published in Fournal of Visualized Surgery. The article has undergone external peer review.

Conflicts of Interest: Both authors have completed the ICMJE uniform disclosure form (available at https://jovs. amegroups.com/article/view/10.21037/jovs.2020.02.07/ coif). The series "Dedicated to the 36th Annual Conference of Japanese Association for Chest Surgery" was commissioned by the editorial office without any funding or sponsorship. The authors have no other conflicts of interest to declare.

Ethical Statement: The authors are accountable for all aspects of the work in ensuring that questions related to the accuracy or integrity of any part of the work are appropriately investigated and resolved. The study was conducted in accordance with the Declaration of Helsinki (as revised in 2013). The study was approved by the institutional review board and the committee of Nippon Medical Chiba Hokusoh Hospital (No. 616). Informed consent to use the data-use agreement including surgical video was obtained from all patients before surgery.

Open Access Statement: This is an Open Access article distributed in accordance with the Creative Commons Attribution-NonCommercial-NoDerivs 4.0 International License (CC BY-NC-ND 4.0), which permits the noncommercial replication and distribution of the article with the strict proviso that no changes or edits are made and the original work is properly cited (including links to both the formal publication through the relevant DOI and the license). See: https://creativecommons.org/licenses/by-nc-nd/4.0/.

\section{References}

1. Gonzalez-Rivas D, Paradela M, Fernandez R, et al. Uniportal video-assisted thoracoscopic lobectomy: two years of experience. Ann Thorac Surg 2013;95:426-32.

2. Ng CS, Kim HK, Wong RH, et al. Single-port videoassisted thoracoscopic major lung resections: experience with 150 consecutive cases. Thorac Cardiovasc Surg 2016;64:348-53.

3. Hirai K, Takeuchi S, Usuda J. Single-port video-assisted thoracic surgery for early lung cancer: initial experience in Japan. J Thorac Dis 2016;8:S344-50.

4. Schuchert MJ, Abbas G, Pennathur A, et al. Sublobar resection for early-stage lung cancer. Semin Thorac Cardiovasc Surg 2010;22:22-31.

5. Hirai K, Usuda J. Surgical technique of right S3 segmentectomy. Asvide 2020;7:076. Available online: http://www.asvide.com/watch/33115

6. Bertolaccini L, Batirel H, Brunelli A, et al. Uniportal video-assisted thoracic surgery lobectomy: a consensus report from the Uniportal VATS Interest Group (UVIG) of the European Society of Thoracic Surgeons (ESTS). Eur J Cardiothorac Surg 2019;56:224-9.

7. Ke H, Liu $\mathrm{Y}, \mathrm{Zhou} \mathrm{X}$, et a 1 . Anterior fissureless uniport vs. posterior intra-fissure triple-port thoracoscopic right 
upper lobectomy: a propensity-matched study. J Thorac Dis 2017;9:3866-74.

8. Hirai K, Enomoto Y, Usuda J. Converting uniportal videoassisted thoracic surgery: multiport or open? J Vis Surg 2019;5:22.

9. Chung JH, Choi YS, Cho JH, et al. Uniportal videoassisted thoracoscopic lobectomy: an alternative to conventional thoracoscopic lobectomy in lung cancer surgery? Interact Cardiovasc Thorac Surg 2015;20:813-9.

doi: 10.21037 /jovs.2020.02.07

Cite this article as: Hirai K, Usuda J. Current status and issues of uniportal video-assisted thoracic surgery in Japan. J Vis Surg 2020;6:27.
10. Hirai K, Usuda J. Uniportal video-assisted thoracic surgery reduced the occurrence of post-thoracotomy pain syndrome after lobectomy for lung cancer. J Thorac Dis 2019;11:3896-902.

11. Yang S, Guo W, Chen X, et al. Early outcomes of robotic versus uniportal video-assisted thoracic surgery for lung cancer: a propensity score-matched study. Eur J Cardiothorac Surg 2018;53:348-52. 
1. Prof. Mrinoshin Okumura: Thoracoscopic view is limited in uniportal VATS. I suppose that flexible thoracoscope is more useful in uniportal procedure. What is your opinion?

\section{Authors' answer:}

In uniportal VATS surgeons, there was no surgeon to recommend strongly to use the flexible scope in present. According to my experience, an operator stands the right side to the patient and face to face position to the assistant. I operate in the bird's eye view to lift the scope perpendicularly to the chest wall. If the surgeon and assistant stand the same side to the patient, it may be useful to secure the surgical view by using the flexible scope.

\section{Prof. Mrinoshin Okumura: Is there any limitation in nodal dissection in uniportal VATS?}

\section{Authors' answer:}

We have difficulty in completing en-bloc lymph node dissection at the same degree with Japanese style that had been carried out at a part of Japanese hospital such as some cancer centers.

However, it is possible to remove the lymph nodes without fail. Two-hand control with operative instruments is still immature for uniportal VATS surgeons. Additional ingenuity and efforts are needed in dissecting lymph node dissection.

New devise and additional ingenuity is under consideration.

3. Prof. Mrinoshin Okumura: What are the reasons for conversion to thoracotomy? Do you convert uniportal VATS directly to thoracotomy, not via multiportal VATS?

\section{Authors' answer:}

As for conversion to thoracotomy or multiport VATS conversion, I have already reviewed in U-VATS lobectomy. I added the related two articles in my manuscript as follows:

"Hirai K, Enomoto Y, Usuda J. Converting uniportal video-assisted thoracic surgery: multiport or open? J Vis Surg 2019;5:22.";

"Chung JH, Choi YS, Cho JH, et al. Uniportal video-assisted thoracoscopic lobectomy: an alternative to conventional thoracoscopic lobectomy in lung cancer surgery? Interact Cardiovasc Thorac Surg 2015;20:813-9.”.

In concrete, the causes of our conversion are mainly as follows:

(I) Related matter of vessel treatment:

Fixed lymph node to the it beginning of A6;

Tumor invasion to PA;

Fall ligated thread from vessel.

(II) Related matter of lung adhesion:

Firm lung adhesion to diaphragm. 